\title{
An evaluation of nanostructured zinc oxide as a fluorescent powder for fingerprint detection
}

\author{
Mi Jung Choi, ${ }^{a}$ Katie E. McBean, ${ }^{\mathrm{b}}$ Ping Hei R. Ng, ${ }^{\mathrm{a}}$ Andrew M. McDonagh, ${ }^{\mathrm{a}^{*}}$ Philip J. \\ Maynard, ${ }^{\mathrm{a}}$ Chris Lennard, ${ }^{\mathrm{c}, \mathrm{d}}$ Claude Roux ${ }^{\mathrm{a}}$ \\ ${ }^{\text {a }}$ Centre for Forensic Science, University of Technology, Sydney, Australia \\ ${ }^{\mathrm{b}}$ Microstructural Analysis Unit, University of Technology, Sydney, Australia \\ ${ }^{\mathrm{c}}$ Forensic \& Technical Services, Australian Federal Police, Canberra, Australia \\ ${ }^{d}$ Current address: National Centre for Forensic Studies, University of Canberra, Australia
}

Email: andrew.mcdonagh@uts.edu.au

Tel: +61295141035 


\begin{abstract}
Zinc oxide is evaluated as a fluorescent powder for the detection of fingermarks on nonporous surfaces. Pure and lithium-doped nanostructured zinc oxide powders were characterized using scanning electron microscopy, x-ray diffraction, and fluorescence spectroscopy. The zinc oxide powders were applied to fresh and aged fingermarks deposited on non-porous surfaces such as glass, polyethylene and aluminium foil. Zinc oxide was found to produce clear fluorescent impressions of the latent fingermarks when illuminated with long-wave UV light.
\end{abstract}

Keywords: Zinc oxide, fingerprints, fingermarks, lithium, fluorescent powder 


\section{Introduction}

We report here the results of our investigations into the use of nanostructured zinc oxide particles as fluorescent powders for latent fingerprint detection.

Zinc oxide $(\mathrm{ZnO})$ powder has been used extensively since the end of the eighteenth century [1], primarily as a white pigment. It has also been used as a base for coloured pigments; for example, with bismuth or cobalt oxide [2,3]. Zinc oxide is also a wide band gap semiconductor and zinc oxide particles typically exhibit photoluminescence in the form of a UV band-edge emission [4]. A broad emission band in the visible region of the spectrum is also observed and arises from intrinsic or extrinsic defects in the material [5].

Powder materials are routinely used in the field of forensic fingerprint detection. The conventional powdering technique involves preferential adherence of powder particles to fingerprint residues to provide contrast between the fingerprint features and the background surface [6]. Zinc oxide has been used in white small particle reagents (SPRs) for fingerprint detection [7]. The small particle reagent technique may be regarded as a wet powdering method where latent fingermarks are visualized using a suspension of fine particles in detergent solution. The technique is effective for the detection of latent fingermarks on wet non-porous surfaces. In each of these applications, the treated fingermarks are visualized using the contrast in colour between the white powder and the background surface.

In the current study, we have explored for the first time the use of the visible luminescence of zinc oxide materials to provide contrast between fingermarks developed with the oxide and the surface substrate. Zinc oxide powders were prepared and applied to latent fingermarks on non-porous surfaces in the form of a small particle reagent or as a powder. The effect of doping the zinc oxide with lithium ions to enhance the visible luminescence was also investigated. 


\section{Experimental}

\section{General}

The following chemicals were purchased from commercial suppliers and used as received: sodium hydroxide (ChemSupply), zinc nitrate hexahydrate (Ajax), lithium chloride (Aldrich), o-xylene (Fluka), Tween 20 (Aldrich). MilliQ water $\left(18 \mathrm{M} \Omega \mathrm{cm}^{-1}\right)$ was used in the synthetic procedures. "Black Emerald” and "Blitz Green” fluorescent magnetic powder were purchased from Lightning Powder, USA.

Secondary electron scanning electron microscope (SEM) images were obtained using a Zeiss Supra 55VP SEM using an in-lens detector. An accelerating voltage of either $5 \mathrm{kV}$ or 10 $\mathrm{kV}$ was used (as indicated in figure captions).

Fluorescence emission spectra of powder samples were recorded on a Varian Cary Eclipse Fluorescence Spectrometer with an excitation slit width of $10 \mathrm{~nm}$ and emission slit width of either $5 \mathrm{~nm}$ or $10 \mathrm{~nm}$. Both excitation and emission filters were used to screen higher order wavelengths.

X-ray diffraction experiments of powder samples were performed using a Siemens D5000 powder diffractometer with a graphite post monochromator with the following parameters: wavelength $1.5406 \AA(\mathrm{Cu} \mathrm{K}-\alpha)$, tube power $1.6 \mathrm{~kW}(40 \mathrm{kV}$ at $40 \mathrm{~mA})$, step size $=0.02$ degrees, time per step $=1 \mathrm{~s}$, divergent slit $=1^{\circ}$, receiving slit $=0.02 \mathrm{~mm}$, scan angle range $=15$ to $75^{\circ}$.

\section{Synthesis}

Zinc oxide particles were prepared by modification of literature procedures [8,9]. Equal volumes $(100 \mathrm{ml})$ of aqueous zinc nitrate $(0.05 \mathrm{M})$ and sodium hydroxide $(0.2 \mathrm{M})$ solutions were prepared. The sodium hydroxide solution was heated to $70{ }^{\circ} \mathrm{C}$ on a hotplate stirrer and the zinc nitrate solution was then added dropwise over $30 \mathrm{~min}$ with stirring. The temperature was kept at $70^{\circ} \mathrm{C}$ and, after $2 \mathrm{~h}$, the white precipitate was collected on a sintered-glass filter 
and rinsed several times with ethanol and then water. The final product was obtained by centrifugation and dried at room temperature. The particles were characterized using scanning tunnelling microscopy, x-ray diffraction and fluorescence spectroscopy.

Lithium-doped zinc oxide particles were prepared using the procedure described above except the zinc nitrate solution was replaced with solutions of the following stoichiometries: (a) $49.5 \mathrm{mM} \mathrm{Zn}\left(\mathrm{NO}_{3}\right)_{2} / 0.5 \mathrm{mM} \mathrm{LiCl}$, (b) $47.5 \mathrm{mM} \mathrm{Zn}\left(\mathrm{NO}_{3}\right)_{2} / 2.5 \mathrm{mM} \mathrm{LiCl}$, (c) $45.0 \mathrm{mM}$ $\mathrm{Zn}\left(\mathrm{NO}_{3}\right)_{2} / 5.0 \mathrm{mM} \mathrm{LiCl}$, and (d) $40.0 \mathrm{mM} \mathrm{Zn}\left(\mathrm{NO}_{3}\right)_{2} / 10.0 \mathrm{mM} \mathrm{LiCl}$.

\section{Development and Imaging of Fingermarks}

All fingermarks were taken from a single donor. Sets of latent fingermarks were deposited in succession after rubbing three fingers over the forehead without recharging with sebum between depositions. For the comparisons on polyethylene and aluminium foil, the centre of the middle fingermark was cut in half. On glass, the middle fingermark was deposited across two adjacent glass slides. For each comparison, half of a fingermark was treated with one reagent and the other half treated with a second reagent. All deposited fingermarks were stored at room temperature. Each treated sample was imaged using a VSC2000/HR Video Spectral Comparator (Foster and Freeman, UK). The sample was illuminated with longwavelength UV light and emission was detected by observation through a $570 \mathrm{~nm}$ long-pass filter.

Powders were applied using a Lightning No.1-0035 fingerprint brush. For small particle reagent, $0.1 \mathrm{~g}$ of zinc oxide powder was added to $10 \mathrm{ml}$ of distilled water and $0.05 \mathrm{ml}$ of Tween 20 surfactant, then the suspension sonicated for $5 \mathrm{~min}$. Samples were immersed in the powder suspension for 2-5 min, depending on the type of surface, and washed with distilled water. The samples were then dried at room temperature prior to imaging. 


\section{Results and Discussion}

\section{Synthesis of zinc oxide particles}

Zinc oxide particles were synthesised using adapted literature procedures [8,9]. Equal volumes of aqueous zinc nitrate $(0.05 \mathrm{M})$ and sodium hydroxide $(0.2 \mathrm{M})$ solutions were mixed and stirred at $70^{\circ} \mathrm{C}$ for $2 \mathrm{~h}$. Lithium-doped samples were prepared by adding lithium chloride to the zinc solution before mixing with the hydroxide solution. As discussed below, the inclusion of lithium significantly affects properties of the nanostructured particles. The powders were characterized by x-ray diffraction measurements, fluorescence spectroscopy, and scanning electron microscopy.

\section{$X$-ray diffraction of powders}

Powder samples were studied by x-ray diffraction measured in the range between 15 and $75^{\circ}$ (see Supporting Information). The data are in good agreement with the data of hexagonal zincite with respect to the position and relative intensity of the peaks [10]. There were no additional XRD peaks associated with the lithium-doped samples compared to the undoped specimens indicating no new crystal-phase formation. This finding is in agreement with recent work investigating $\mathrm{ZnO}$ nanorods doped with lithium up to a concentration of $10 \%$ [11]. The structural agreement between the doped and pure $\mathrm{ZnO}$ patterns indicates that each of the dopant atoms do not significantly alter the packing structure of the parent $\mathrm{ZnO}$ lattice although as shown below, some interesting morphological differences between doped and undoped are observed in the scanning electron microscope images. 


\section{Scanning electron microscopy}

The micro- and nanostructure of the zinc oxide particles was investigated using a scanning electron microscope (SEM). Figure 1A shows an SEM image of undoped-zinc oxide particles. The images reveal flower-like zinc oxide particles that are generally in the size range $1-3 \mu \mathrm{m}$ with a reasonably uniform size distribution.

Figures 1B \& $\mathrm{C}$ shows higher magnification images that reveal the nanostructure of the micron-sized particles. Well-oriented, flower-like structures are evident with each "petal" composed of many small nanoparticles. A similar morphology of zinc oxide has been previously reported [12-14].

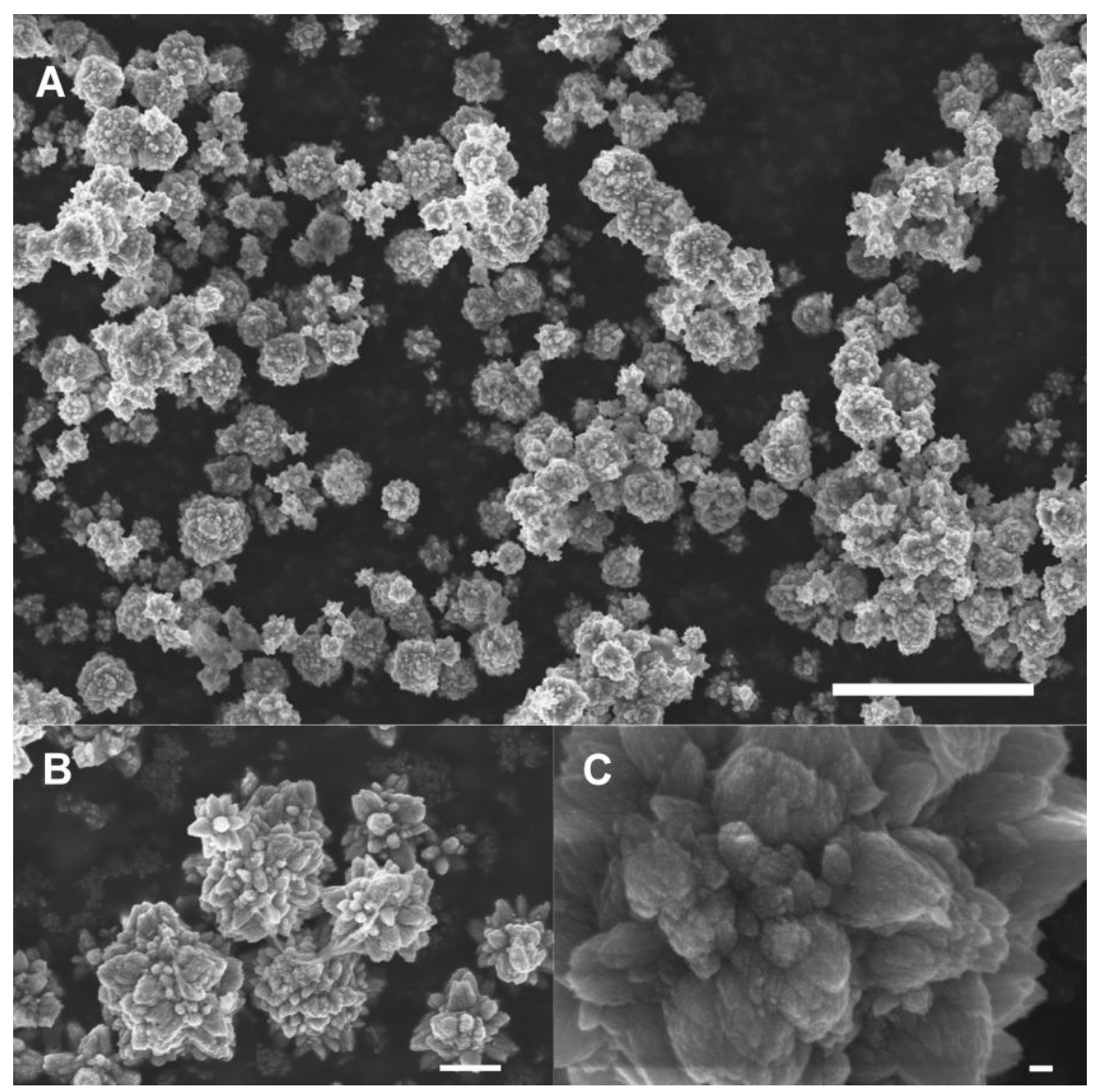

Fig. 1: A: Scanning electron microscope image of undoped zinc oxide particles (5000x magnification, accelerating voltage $=10 \mathrm{kV}$ ). Scale bar $=10 \mu \mathrm{m}$. B: Image at 31200x magnification, scale bar $=1 \mu \mathrm{m}$ (accelerating voltage $=5 \mathrm{kV})$. C: Image at $100,000 \mathrm{x}$ magnification, scale bar $=200 \mathrm{~nm}($ accelerating voltage $=10 \mathrm{kV})$. 
When lithium ions were added to the reaction mixture, more plate-like "petal" structures were observed in the SEM images. The mean diameter of the particles obtained from addition of $1 \%$ lithium (relative to zinc) is approximately $1-2 \mu \mathrm{m}$, similar to the undoped samples. Upon increasing the ratio of lithium to zinc in the reaction mixture, more needle-like structures were observed on the surfaces of the isolated powders. Figure 2 shows SEM images of lithium-doped zinc oxide particles from reactions where a $10 \% \mathrm{Li}: \mathrm{Zn}$ ratio was used (Figure2A \& B, and a 20\% Li:Zn ratio (Figure 2C). In each case, the particle diameter was approximately 1-2 $\mu \mathrm{m}$. Recently, Zhu et al [15] proposed that the presence of lithium dopants in zinc lattice sites will decrease the number of inherent oxygen vacancies. This in turn should promote the growth of more ordered structures. This effect is observed in the images shown in Figure 2 where an increase in well-oriented growth occurs with increasing lithium concentration. In general, it is apparent that the addition of lithium ions has a significant effect on the morphology of the resultant nanostructured zinc oxide particles although as mentioned above, this is not directly evident from powder XRD data. 


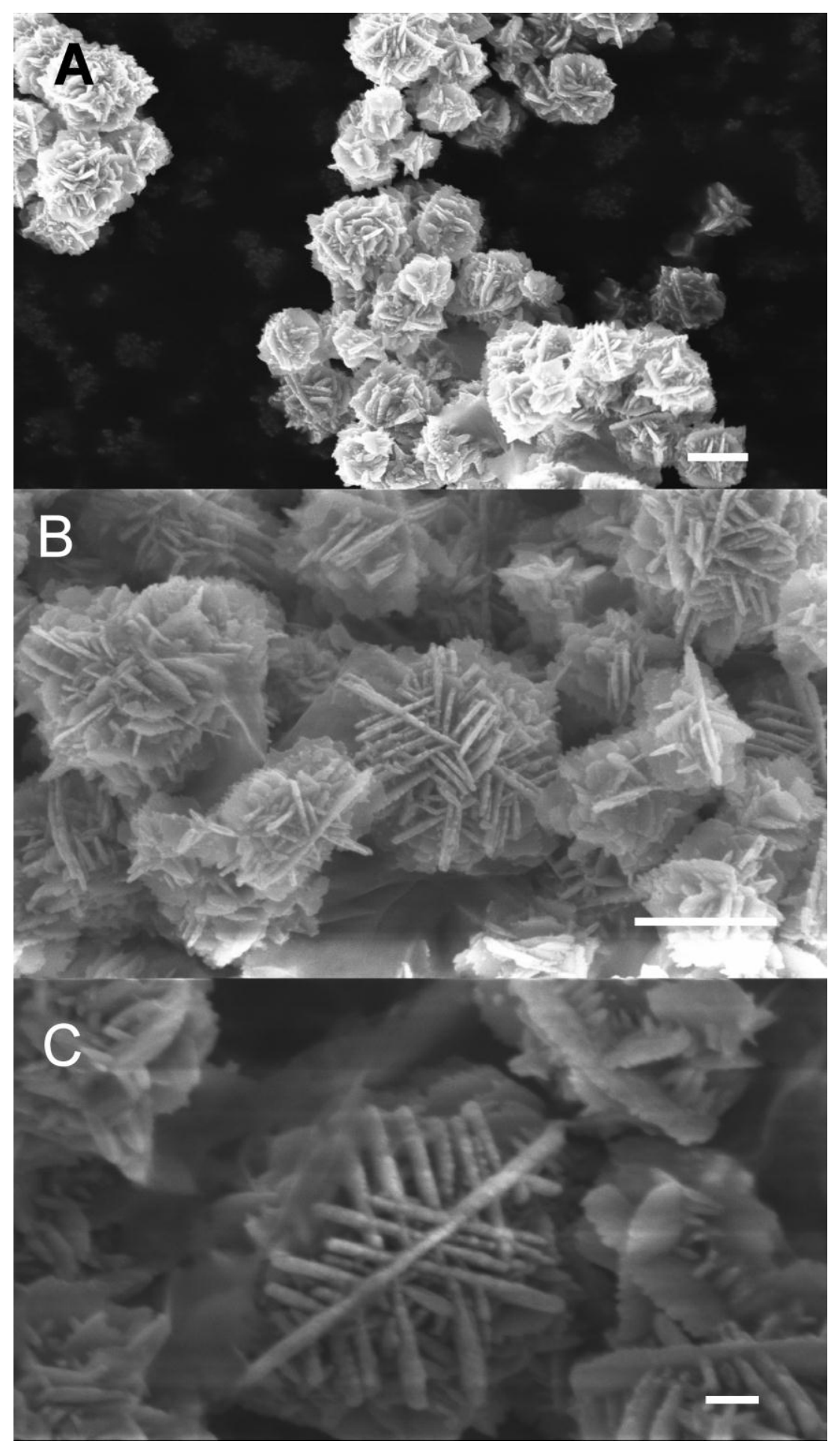

Fig. 2. SEM images of lithium-doped zinc oxide particles. A \& B: 10\% Li-doped; scale bar = $1 \mu \mathrm{m}$ (accelerating voltage $=10 \mathrm{kV}) . \mathrm{C}: 20 \%$ Li-doped; scale bar $=200 \mathrm{~nm}$ (accelerating voltage $=5 \mathrm{kV})$. 


\section{Fluorescence Spectroscopy}

Figure 3 shows the fluorescence spectrum obtained from undoped, dried zinc oxide powder. It is typical of zinc oxide powder with a relatively intense emission at $388 \mathrm{~nm}$ and a broad emission band centred at $\sim 587 \mathrm{~nm}$. The peak at $388 \mathrm{~nm}$ is the near band-edge emission (NBE) and corresponds to the band-gap energy of this compound (3.3 eV at room temperature). The green emission band at $\sim 587 \mathrm{~nm}$ is attributed to defects such as oxygen vacancies [5].

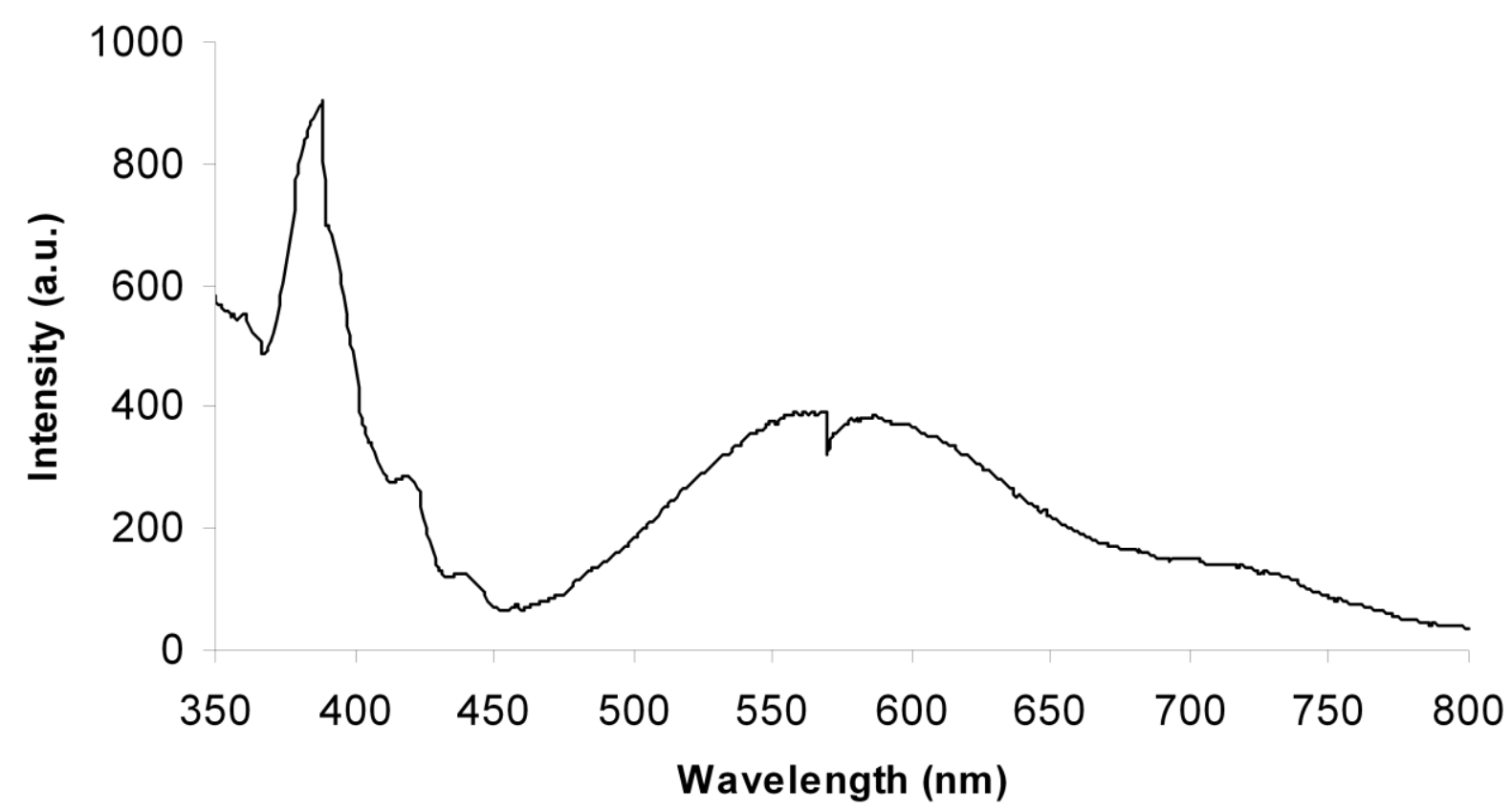

Fig. 3. Fluorescence emission spectrum of $\mathrm{ZnO}$ powder. Excitation wavelength $=320 \mathrm{~nm}$, excitation slit width $=10 \mathrm{~nm}$ (features at 460 and $570 \mathrm{~nm}$ are artefacts arising from filter changes).

In this work, we sought to exploit the visible luminescence of zinc oxide to enable detection of latent fingermarks on various surfaces. It was anticipated that incorporation of lithium ions into the material would increase the intensity of the luminescence band in the visible region by introducing more surface defect sites [16]. As described above, the inclusion of different amounts of lithium ions in the reaction mixtures had an observable effect on the 
particle surface morphology by SEM analysis. Figure 4 shows the effect of lithium ion concentration on the fluorescence emission spectra of lithium-doped zinc oxide samples. This data suggests that a dopant concentration of $\sim 10 \%$ (relative to zinc) is optimal to enhance the visible emission.

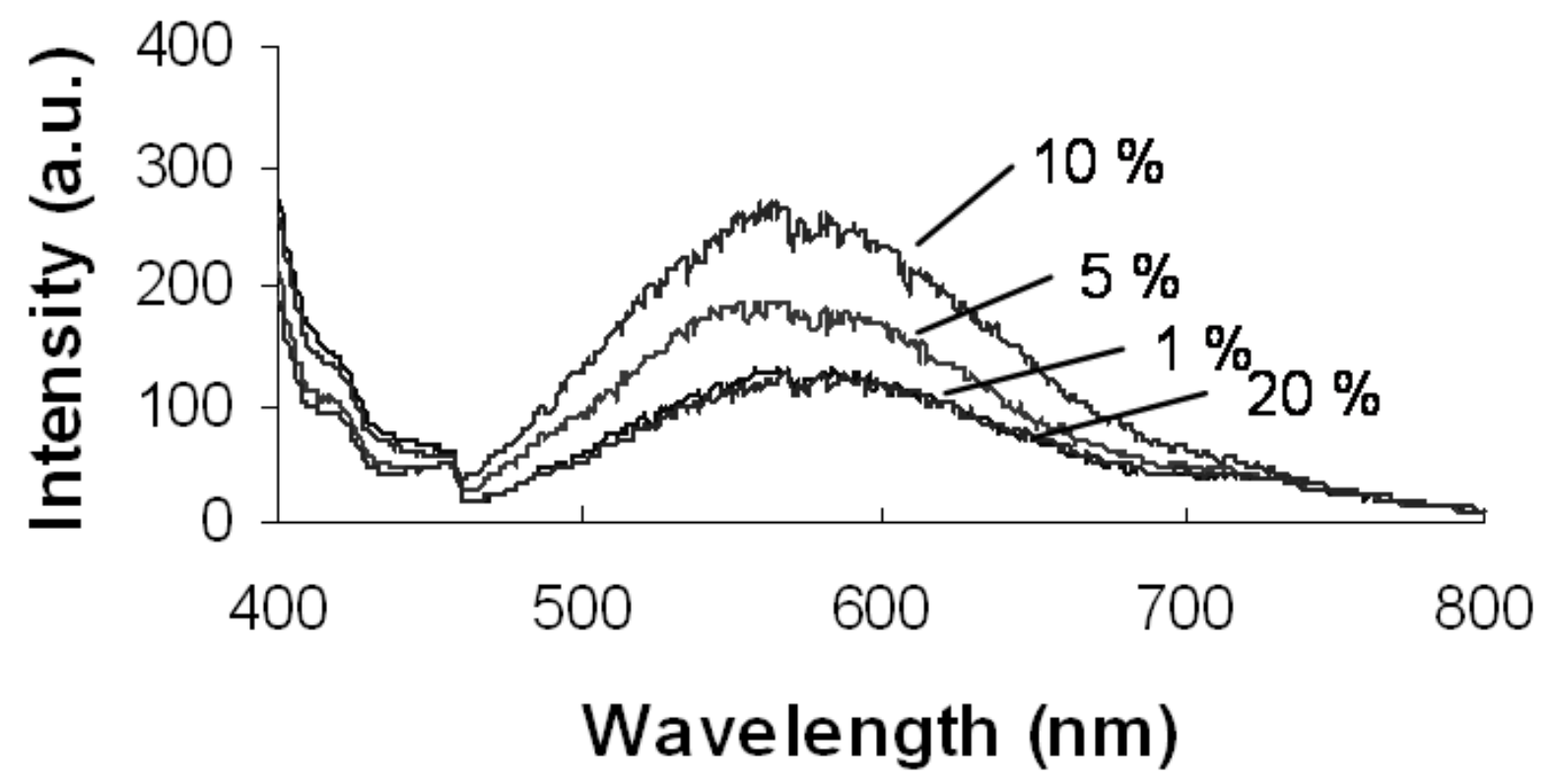

Fig 4. Fluorescence emission spectra for $\mathrm{ZnO}$ doped with 1\%-20\% Li. Excitation wavelength $=360 \mathrm{~nm}$, excitation slit width $=5 \mathrm{~nm}$.

Because the luminescence properties of nanosized zinc oxide particles depend greatly on their surface properties [17], experiments were performed to investigate the effect of mechanical grinding on the fluorescence of the particles. It is apparent from the fluorescence emission spectra of $10 \%$ lithium-doped zinc oxide particles before and after grinding (see Supporting Information) that the defect fluorescence band at $\sim 587 \mathrm{~nm}$ decreases after grinding of the sample while the intrinsic emission band at $\sim 380 \mathrm{~nm}$ increases. Similar behaviour was observed for undoped zinc oxide powders indicating that the inclusion of lithium ions is not responsible for the spectroscopic changes upon mechanical grinding. A recent study [18] reported a similar decrease in the visible luminescence excited by UV light 
after grinding experiments and suggested that mechanical grinding induces changes in the defect structure of the zinc oxide crystal lattice. Our results are consistent with these findings but, in addition to the decrease in defect emission, we note the concomitant increase in the band gap emission intensity.

From the fluorescence emission spectroscopy, we conclude that the powders are fluorescent in a region suitable for use in latent fingerprint detection. Doping with lithium ions somewhat enhances the intensity of the visible emission band while mechanical grinding has the opposite effect.

\section{Application to latent fingermark detection}

Zinc oxide powders were applied to fingermarks on non-porous surfaces such as glass, polyethylene, and aluminium foil. The powders were applied by the conventional powder and brush method and as a small particle reagent (SPR). In the SPR method, zinc oxide powder was dispersed in distilled water containing a surfactant (Tween 20). The specimens were immersed in the suspension, washed with distilled water, and then dried at room temperature before imaging.

Figure 5 shows a ten-day-old fingermark deposited on glass that has been developed with undoped zinc oxide using the powder and brush technique. The specimen was illuminated at $350 \mathrm{~nm}$ and it is apparent that the luminescence of the zinc oxide provides good contrast between the fingerprint ridges and the background. 


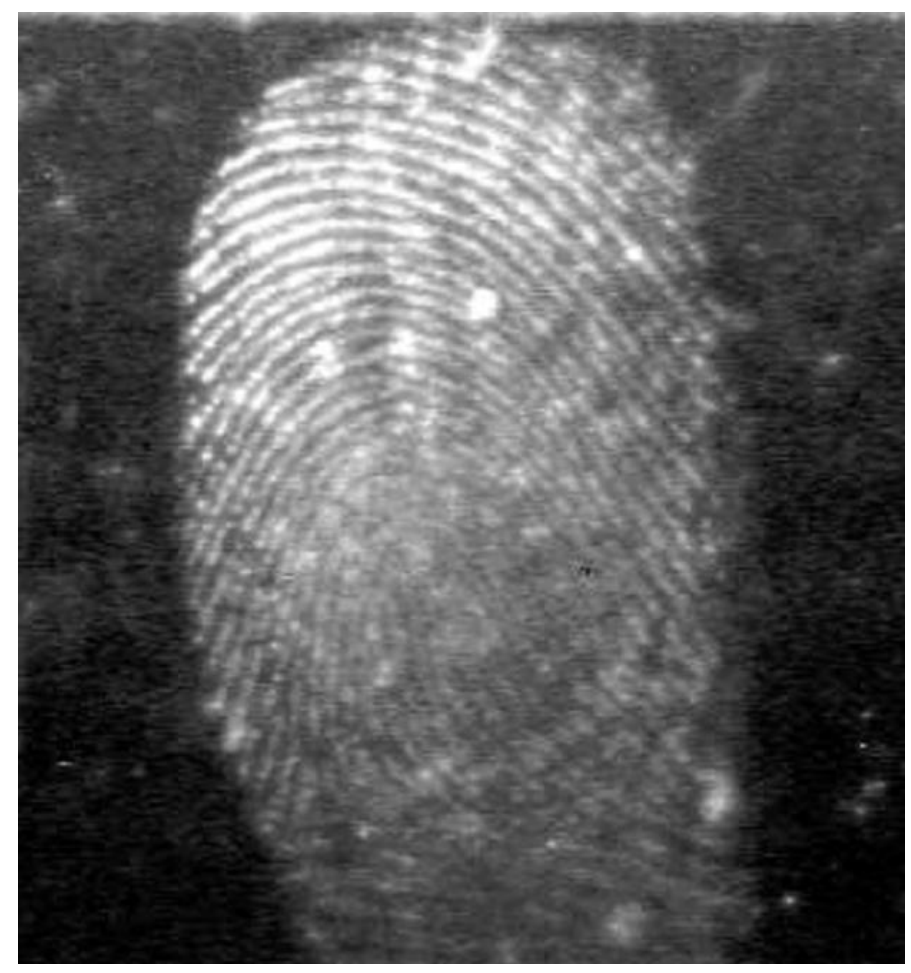

Figure 5: A ten-day-old fingermark on glass developed with nanostructured $\mathrm{ZnO}$ using the powder and brush technique and detected using a $570 \mathrm{~nm}$ long pass filter with $350 \mathrm{~nm}$ illumination.

Figure 6 shows a comparison of fingermarks on polyethylene developed using zinc oxide by two different techniques. The left half of this fingermark set shows the results of treatment using the powder and brush technique, while the right half shows fingerprints developed using SPR. It was found that SPR consistently yielded well-developed fingermarks even from older prints. The powder and brush technique often resulted in significant background staining. A comparison of the two techniques on aluminium foil showed that both powdering and SPR yielded excellent results on this surface. We suggest that an electrostatic effect exists with dry powder on plastic, but this does not occur with SPR nor on aluminium foil. 


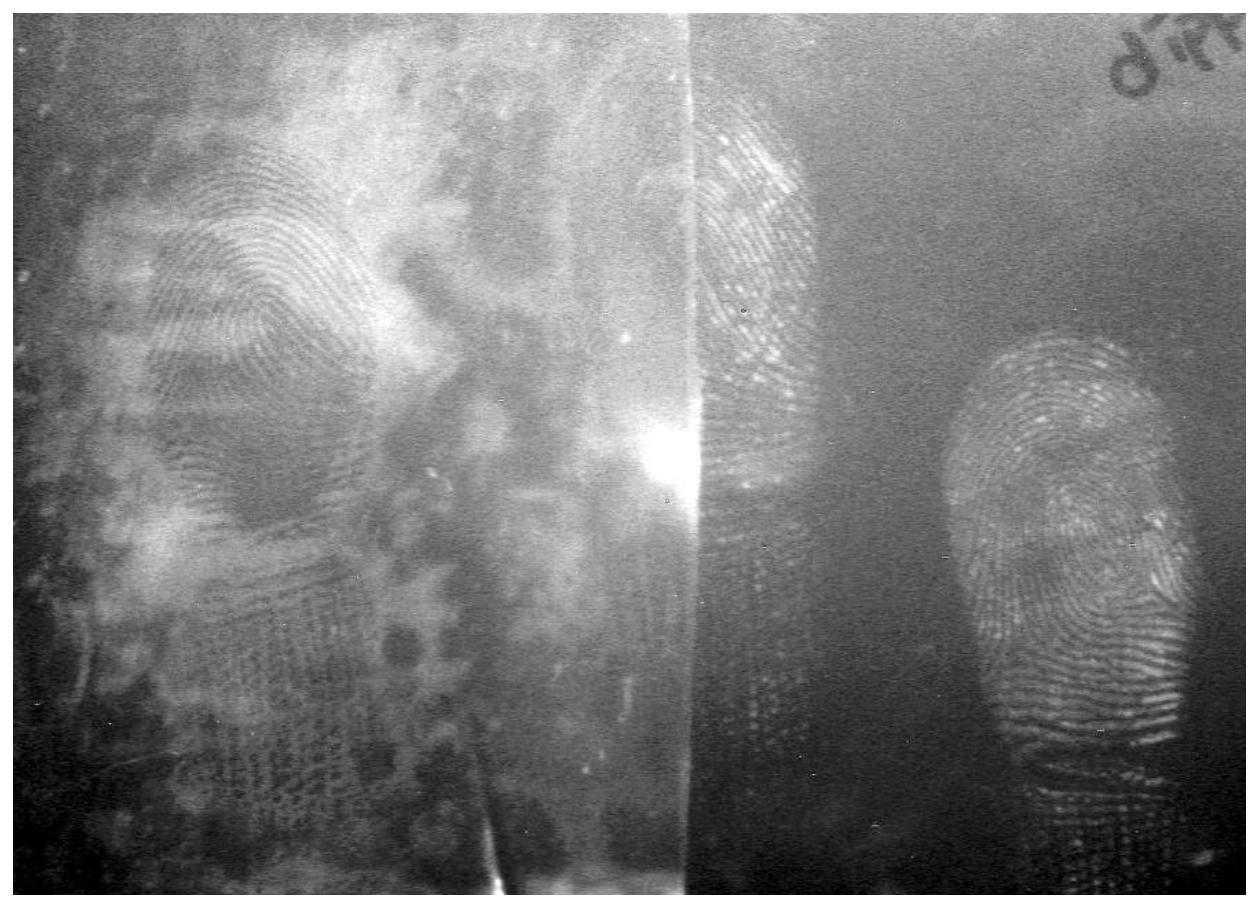

Figure 6. Three-week-old fingermarks on polyethylene, developed using powdering (left) and SPR (right); illumination at $350 \mathrm{~nm}$ and detection using a $570 \mathrm{~nm}$ long-pass filter.

Figure 7 shows a comparison of fingermarks treated with undoped zinc oxide and with $10 \%$ Li-doped zinc oxide powders using the SPR technique. Although the doped zinc oxide powder yields slightly more intense images, we generally observed little significant difference in intensity or detail between the two treatments, suggesting that the benefits of doping with lithium may be minimal in a practical sense. The handling properties of the Li-doped zinc oxide powder were similar to the undoped powder. 


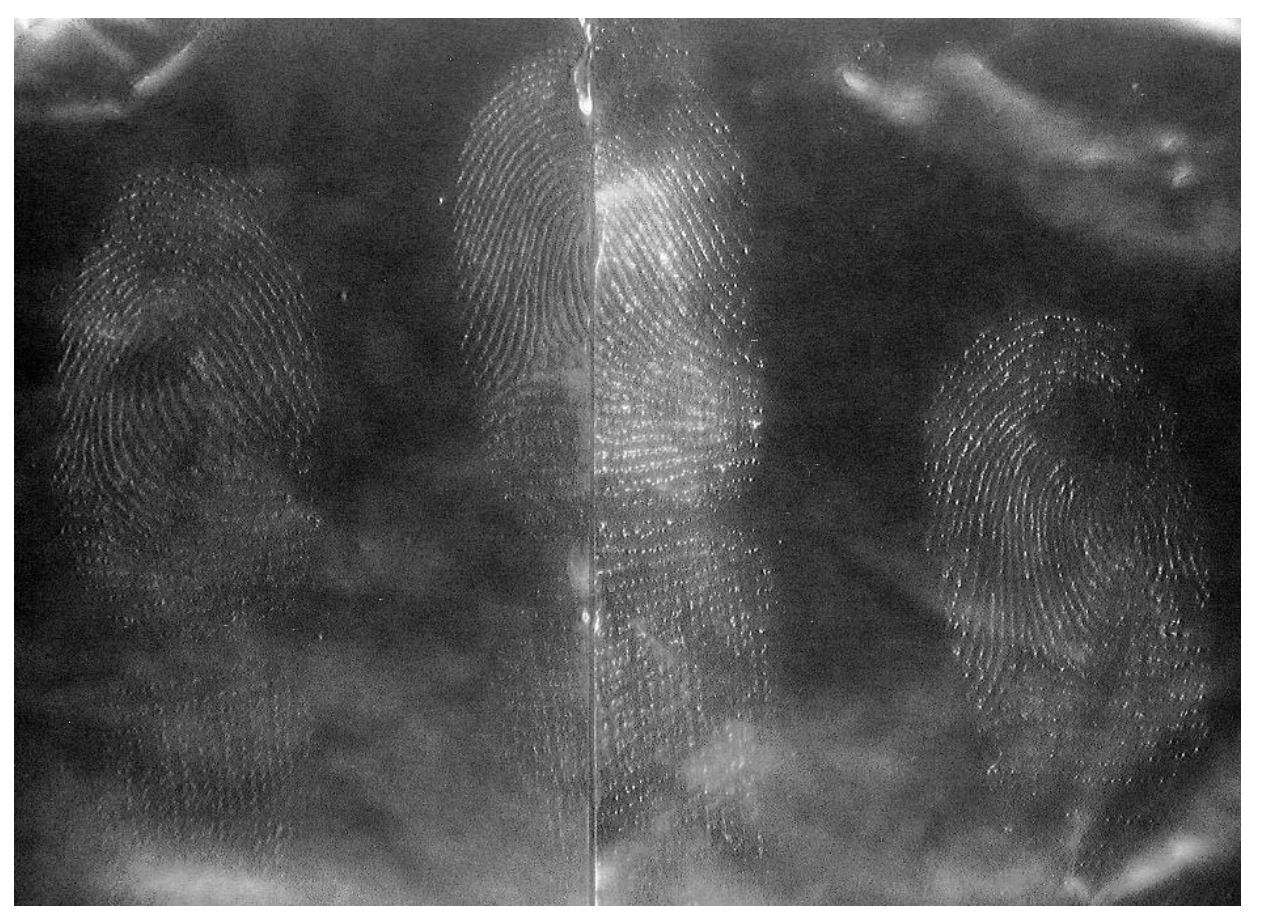

Figure 7. Fresh fingermarks on aluminium foil treated with $\mathrm{ZnO}$ (left) and $\mathrm{ZnO}: \mathrm{Li} 10 \%$ (right); illumination at $350 \mathrm{~nm}$ and detection using a $570 \mathrm{~nm}$ long-pass filter.

Development of fingermarks using the powder and brush technique generally deposited more zinc oxide material on fresh fingermarks on all of the tested surfaces. On polyethylene surfaces, the powder and brush technique often resulted in background staining. SPR using zinc oxide pigment gave excellent results in all cases.

Experiments were performed to compare $\mathrm{ZnO}$ with some commercial fingerprint powders on aluminium surfaces. "Black Emerald" and "Blitz Green" fluorescent magnetic powder were used in a comparison of $10 \% \mathrm{Li}$ doped $\mathrm{ZnO}$ particles (see Supporting Information for images). When observed under white light, very similar development of fingermarks was observed. With UV illumination, the $\mathrm{ZnO}$ particles yielded significantly less fluorescence intensity than the commercial powders although the fingermarks developed with zinc oxide still showed excellent ridge detail with minimal background staining.

In summary, the zinc oxide particles applied to latent fingermarks produced clear fluorescent impressions when illuminated with long-wave UV light. In comparison with some 
commercially available fluorescent fingerprint powders, the fluorescence intensity was somewhat lower for the zinc oxide powders described here but it produced less background development, which may lead to a better overall contrast. Other advantages include a straightforward synthesis from relatively cheap starting materials, low toxicity, and the utility of using the material as a powder or as a wet small particle reagent.

\section{Conclusion}

Nano-structured zinc oxide powders can provide sharp and clear development of latent fingermarks with minimal background staining on non-porous surfaces. Scanning electron microscope images showed that the nanostructure of these particles is affected by the addition of lithium ions to the reaction mixture. The synthesized zinc oxide exhibited emission peaks centred at approximately 380 and $587 \mathrm{~nm}$.

Zinc oxide applied to fingermarks produced fluorescent fingermarks under long-wave UV illumination and can be used as a powder or small particle reagent. The SPR was significantly more effective on polyethylene and on aged prints than the dry powder technique. A comparison between pure zinc oxide and Li-doped zinc oxide showed no significant differences under either UV or white light illumination even though fluorescence spectroscopy showed the Li-doped material to be somewhat more fluorescent.

\section{References}

1. Eastaugh N, Walsh V, Chaplin T, Siddall R, Pigment Compendium: A Dictionary of Historical Pigments, Elsevier Butterworth-Heinemann, 2004.

2. Šulcová P, Trojan M (1998) Dyes Pigments 36:287

3. Šulcová P, Trojan M (1999) Dyes Pigments 40:83.

4. Djurišić AB, Leung YH (2006) Small 2:944.

5. Djurišić AB, Leung YH, Tam KH, Ding L, Ge WK, Chen HY, Gwo S (2006) Appl. Phys. Lett. 88:103107.

6. Champod C, Lennard C, Margot P, Stoilovic M, Fingerprints and Other Ridge Skin Impressions, CRC Press, 2004.

7. Cucè P, Polimeni G, Lazzaro AP, De Fulvio G (2004) Forensic Sci. Int. 146S:S7. 
8. Wu C, Qiao X, Chen J, Wang H, Tan F, Li S (2006) Material Lett. 60:1828.

9. McBean K, Philips M, Goldys E (2006) Microsc. Microanal. 12:327.

10. International Centre for Diffraction Data, Powder Diffraction File (PDF) Version 2004.

11. Height MJ, Mädler L, Pratsinis SE, Krumeich F. (2006) Chem. Mater. 18:572.

12. Chittofrati A, Matijevic E, (1990) Colloids and Surfaces, 48:65.

13. Zhang J, Sun L, Yin J, Su H, Liao C, Yan C, (2002) Chem. Mater. 14:4172.

14. Wang J, Cao J, Fang B, Lu P, Deng S, Wang H, (2005) Material Lett. 59:1405.

15. Zhu XW, Li YQ, Lu Y, Liu LC, Xia YB, (2007) Mater. Chem. Phys. 102:75.

16. Sann J, Hofstaetter A, Pfisterer D, Stehr J, Meyer BK (2006) Phys. Stat. Sol. (C) 3:952.

17. Sakohara S, Ishida M (1998) J. Phys. Chem. B. 102:10169.

18. Śćepanović M, Srećković T, Vojisavljević K, Ristić MM (2006) Science of Sintering 38:169. 